PLANT DEVELOPMENT

\section{Fighting for space}

Nature 522, 439-443 (2015)

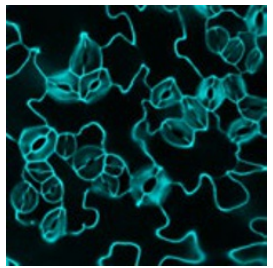

Stomata are microscopic pores on the plant epidermis that mediate gas exchange while minimizing water loss. Genetic studies have identified a peptide from the epidermal pattern factor family, EPF2, that represses stomatal development and an EPF-like peptide, STOMAGEN, that promotes stomatal development. EPF2 is secreted from the stomatal lineage cells and interacts with the ERECTA (ER) family of leucine-rich repeat receptor kinases (ER, ERL1 and ERL2) and the co-receptor TOO MANY MOUTHS (TMM), which are expressed in the surrounding cells. This activation of these receptors was thought to prevent the ectopic formation of stomata. STOMAGEN is secreted by cells underlying the epidermis, but the mechanism by which STOMAGEN promotes stomatal development was still unclear. One possibility was that STOMAGEN and EPF2 might compete for access to the ER family of receptors. To test this model, Lee et al. performed a series of genetic experiments and found that overexpression of STOMAGEN, which normally increases stomatal number, retained activity unless all ER receptors were mutated, suggesting that STOMAGEN required the ER receptors for activity. Consistent with this, co-immunoprecipitation experiments revealed that STOMAGEN directly interacts with all ER receptors and TMM. The authors used a quartz crystal microbalance (QCM) biosensor platform to examine STOMAGEN and EPF2 binding to ER and TMM and found that both peptides bound to each receptor with similar affinity. The authors confirmed the theory that STOMAGEN and EPF2 might compete for access to ER by showing that increasing the amounts of STOMAGEN relative to EPF2 blocked EPF2ER binding and reduced EPF2-mediated inhibition of stomatal development. These findings should inspire further studies to determine whether changes in ligand concentration or receptor sensitivity dictate stomatal formation during plant development.
PHOTOPHARMACOLOGY

\section{Microtubules see the light}

Cell doi:10.1016/j.cell.2015.06.049
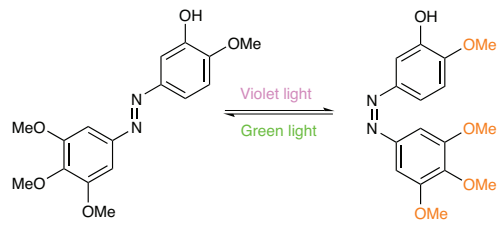

Microtubules are dynamic tubulin polymers in the cytoskeleton that serve as conduits for cellular transport and scaffolds for cellular motility. Microtubules are also critical components of the mitotic spindle required for chromosomal segregation during cell division. Thus, inhibitors of microtubule assembly and dynamics, such as colchicine or paclitaxel, are potent mitotic poisons with antiproliferative properties. Because their low specificity can lead to systemic toxicity, improved approaches to targeting the delivery or bioactivity of these compounds could enhance their utility as probes of microtubule biology and their selectivity as targeted chemotherapeutics. Borowiak et al. now report a photopharmacological approach for the reversible small-molecule control of microtubule dynamics. Building on their earlier successes with light-induced gating of ion channels, the authors surmised that they could achieve light control of microtubule dynamics by replacing the central carboncarbon bond of combretastatin A-4, a colchicine analog, with an azobenzene moiety that enables light-dependent isomerization between an active cis form, which assembles the tetramethoxyaryl pharmacophore, and an inactive trans form. They synthesized a series of these 'photostatins', and demonstrated that violet light induces the active cis conformation, whereas green light switches to the inactive trans conformation in a highly reversible and durable manner induced by low-intensity light. Furthermore, in the dark, the system decays to the more stable trans photostatin by providing an 'off'-state background of bioactivity. Biochemical assays demonstrated that cis-photostatins compete for the colchicine-binding site on tubulin and inhibit tubulin polymerization in vitro. Across several cell types, photostatins display 250 -fold greater cytotoxicity under violet light illumination than in the dark, inducing mitotic arrest and apoptotic cell death. The photostatins were used to toggle microtubule dynamics inside living cells, to pause and reorient embryonic development in Caenorhabditis elegans, and to perturb the cytoskeleton in live mice by local tissue illumination. This unique system offers a useful tool for probing mechanistic questions in microtubule biology with spatial and temporal control, and also shows promise for tumor-specific chemotherapy, by localizing the cytotoxicity of antimitotic poisons within light-targeted tumor tissues.

TLS

PROTEIN PHOSPHORYLATION

\section{KISSing kinases}

J. Cell Biol. 209, 895-912 (2015)

Rho-associated kinase (Rho kinase) regulates different cellular functions ranging from migration to adhesion through the phosphorylation of substrate proteins such as myosin light chain and tau. Although genetic and biochemical studies have identified a number of Rho kinase substrates, many potential substrates still remain uncharacterized. To ensure a more comprehensive identification of Rho kinase targets, Amano et al. developed a method called kinase-interacting substrate screening (KISS) that involved incubating brain lysates with affinity beads coated with the catalytic domain of Rho kinase in the presence or absence of ATP. These samples were run through a titanium oxide column to enrich for phosphorylated peptides, which were then analyzed by LC/MS/MS. Overall, the authors identified a total of 140 proteins containing 356 phosphorylation sites comprising known and novel Rho kinase substrates. In particular, they focused on the planar polarity protein Scribble (Scrib), which was phosphorylated at two sites in the C-terminal domain by Rho kinase. Additional proteomic studies found that a known Rho kinase interactor, Shroom2, interacted with the C-terminal domain of Scrib in a phosphorylationdependent manner. Scrib, Rho kinase and Shroom 2 formed a tertiary complex that was dependent on Scrib phosphorylation. Given that interactions between Shroom 2 and Rho kinase were associated with actomyosin contractility, the authors wanted to confirm whether Scrib was also involved in this process. Scrib was co-localized at the cellular edge with Shroom 2 and Rho kinase, where there was increased contractility; knockdown of Scrib blocked this activity. Finally, the authors were able to apply their KISS method to identify kinase substrates of nine different protein kinases, including PKA and MAPK1. The planned deposition of these findings into a public database will provide a great resource for researchers seeking to identify particular substrates that are required for a kinasedependent activity.

Written by Mirella Bucci, Catherine Goodman Stéphane Larochelle, Grant Miura and

Terry L. Sheppard 